\title{
Tiny new oncogenes
}

DOI:

10.1038/nrc1902

URLs

P53

http://www.

ncbi.nlm.nih.

gov/entrez/query.

fcgi? $\mathrm{db}=$ gene $\& \mathrm{~cm}$

d=Retrieve\&dopt

=full report\&list

uids $=7157$

CDK2

http://www.

ncbi.nlm.nih.

gov/entrez/query.

fcgi? $\mathrm{db}=$ gene $\& \mathrm{~cm}$

d=Retrieve\&dopt

=full_report\&list

$\underline{\text { uids }=1017}$

LATS2

http://www.

ncbi.nlm.nih.

gov/entrez/query.

fcgi? db=gene\&cm

$\mathrm{d}=$ Retrieve\&dopt

$=$ full report\&list

uids $=26524$
A new screen for the function of human microRNAs (miRNAs) has implicated two of them as oncogenes. Reuven Agami and colleagues have shown that miR-372 and miR-373 co-operate with oncogenic Ras to overcome the need for p53 loss in the transformation of cells, and that this occurs in some testicular germ-cell tumours.

The authors created a library of vectors that expresses most cloned human miRNAs, and also constructed an array with which to detect the expression of the members of this library. They then virally transduced cultured fibroblasts with both their library and oncogenic Ras. Cells with wild-type $\mathrm{p} 53$ respond to oncogenic Ras by senescing, and the loss of p53 is usually needed to overcome this antiproliferative response. However, the expression of two miRNAs - miR-372 and miR-373 (which are homologues of each other) - allowed the cells to continue proliferating.

These results were independently validated, and further study showed that cells transduced with these miRNAs were less likely to have a senescent phenotype and had a proliferative advantage even without oncogenic Ras.

To test the mechanism by which this occurs, the authors looked at the levels and activity of p53 in these transformed cells, but found them unchanged. Instead, they found that the activity of cyclin-dependent kinase 2 (CDK2) was not reduced. Normally, the activation of p53 in response to oncogenic Ras leads to the inhibition of CDK2, but this can be relieved by miR-372 and miR-373. So, what are the direct targets of the miRNAs that mediate this effect?
The genes that were found to be downregulated by the miRNAs in a microarray study were analysed for miRNA-target sequences. The most promising candidate was large tumour-suppressing homologue 2 (LATS2). An immunoblot assay verified that LATS2 is a target of the miRNAs, and luciferase assays with the $3^{\prime}$ untranslated region of the gene showed that LATS2 is directly downregulated by the two miRNAs. This might be the first step towards a full mechanistic explanation of the phenomenon.

Do miR-372 and miR-373 have a role in the development of tumours in patients? The authors looked for miR-372 and miR-373 expression in primary cells and cell lines from testicular germ-cell tumours, as these are predominantly p53-positive. Most samples expressed the miRNAs, and all of those that did contained wild-type p53. Interestingly, two of the four tumours that did not express the miRNAs had inactivating mutations in the TP53 gene, an extremely rare event in this type of tumour. By contrast, expression of the miRNAs was rare in somatic-cell tumours.

These results demonstrate a new mechanism for bypassing senescence in 553 wild-type tumours. It remains to be seen how widespread oncogenic miRNAs are, but the study has also provided a system for uncovering the roles of other miRNAs in tumorigenesis.

Patrick Goymer

ORIGINAL RESEARCH PAPER Voorhoeve, P. M et al. A genetic screen implicates miRNA-372 and miRNA-373 as oncogenes in testicular germ cell tumours. Cell 124, 1169-1181 (2006) FURTHER READING Esquela-Kerscher, A. \& Slack, F. J. Oncomirs - microRNAs with a role in cancer. Nature Rev. Cancer 6, 259-269 (2006)

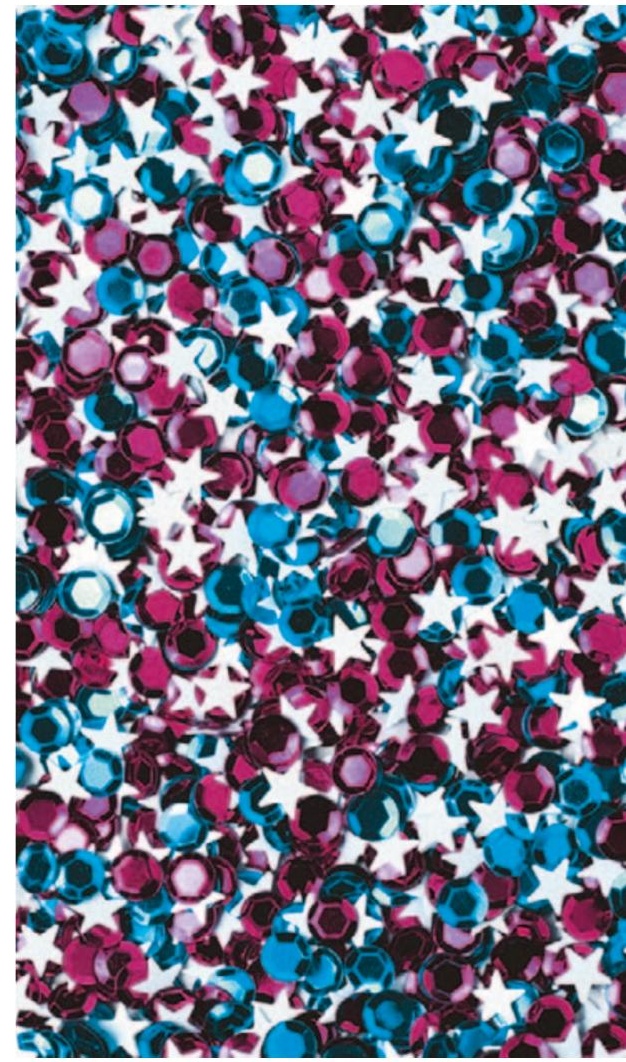

